\title{
Solid lipid nanoparticle of hesperidine exerts diethylnitrosamine/phenobarbital-induced hepatocellular carcinoma via alteration of PI3K/Akt pathway
}

Prakash BHATT* $^{*}$

Biotechnology, Fermentis, India

Introduction: Hepatocellular carcinoma (HCC) is the most frequently known liver disease and the third most common cause of tumour death in the world. Researchers targeted the pathways of phosphatidylinositol-4, 5-bisphosphate 3-kinase/protein kinase B and mitogen activated protein kinase to inhibit the proliferation and development of cells. In this analysis, the solid lipid nanoparticle (SLN) of hesperidine (HP) was manufactured and scrutinized against HCC induced by diethylnitrosamine (DEN) by altering the PI3K/Akt pathway.

Methods: For the preparation of HP-SLN, the double emulsion solvent displacement method was used and DEN (200 mg/kg) and phenobarbital ( $8 \mathrm{mg} / \mathrm{kg}$ ) was used for the induction of HCC. Estimates were given for hepatic, non-hepatic, antioxidant, pro-inflammatory and inflammatory cytokines. The genetic effects of HP-SLN on Pdk1, Akt1, Pik3r1, Map3k1, Erbb2, PIk3ca were assessed using semiquantitative RT-PCR analysis. Morphological and histopathological hepatic tissue components were estimated.

Results: Surface methodology suggests the $174.5 \mathrm{~nm}$ particle size and 0.340 polydispersity index for HP-SLN. HP-SLN significantly $(p<0.001)$ reduced the hepatic nodules $(72 \%)$ and enhanced the body weight $(48.3 \%)$; reduced hepatic nodules $(83.3 \%)$. HP-SLN significantly $(p<0.001)$ modulated the hepatic parameter viz., AFP (78.3\%), AST (61.3\%), ALP (60.3\%), ALT (55.8\%), CEA (58.3\%), GGT (63.2\%); non-hepatic parameter viz., albumin (52.5\%), total protein (63.4\%), BUN (54.6\%), bilirubin (67.3\%), direct bilirubin (52.3\%); antioxidant.

Conclusions: In conclusion, HP-SLN regulated the Akt and PI3K pathways involved in suppression of hepatic cancer growth and proliferation and suggest the chemoprotective effect. 